\title{
Floating Content: Experiences and Future Directions
}

\author{
Shahzad Ali \\ Department of Computer Science \\ Jouf University, Tubarjal, Kingdom of Saudi Arabia
}

\begin{abstract}
Floating content is a promising communication paradigm based on pure ad hoc communications. It has a huge usage potential for various context-aware applications. In this paper, recent research related to floating content communication paradigm is presented. This paper focuses on some of the vital experiences ranging from analytical models to simulations to the real-world implementations. Some important results on the performance of floating content based on analytical models, simulations, and real-world implementations are presented. These results not only show the usefulness of the existing analytical models but also explain the ways of extending these existing models for incorporating new communication technologies and mobility models. This paper also highlights the energy consumption of smartphone applications based on floating content and explains how new communication technologies impact the feasibility of using floating content as a communication service for different applications. Based on the experiences, new future directions are highlighted that can prove to be very beneficial for researchers investigating this area.
\end{abstract}

Keywords_Floating content; opportunistic communications

\section{INTRODUCTION}

A massive growth in mobile computing and an abundance of smart devices are consistently driving applications toward context-awareness [1]. Location is the most common type of context used in such applications. Mostly such context-aware applications deal with data having spatial and temporal constraints. For example, a location-aware parking finding application makes use of information about a free parking slot that is available for a limited time and is of interest to the people searching for parking location nearby. Opportunistic communications due to their specific characteristics can prove to be very beneficial for such applications. Several opportunistic communication paradigms are available in literature under different names [2] [3] [4]. They all aim at making a piece of information available over a restricted geographic area. The term "floating content" was introduced in [3]. Fig. 1 illustrates the basic working of floating content service. For the implementation of floating content service, it is assumed that all nodes rely on pure ad hoc communications (Bluetooth, WiFi-Direct, etc.) for content transfer and there is no fixed infrastructure like WiFi or cellular network availability. As the first step for floating content, a node creates a piece of content and defines a range called "Anchor Zone" for that content. Anchor zone refers to a geographical area in which the content is replicated using ad hoc communications whenever two nodes come in communication range of each other. Therefore, nodes within an anchor zone keep on replicating the generated content and in this way that content "floats" within the anchor zone. Once a node goes out of the anchor zone, it deletes the content. This mechanism is illustrated in Fig. 1. In this way a new node entering the anchor zone has an opportunity for getting the content if it comes in contact of a node already possessing the content. This is called the success ratio and it is one of the fundamental performance metrics for floating content. Formally, success ratio can be defined as the average fraction of nodes getting content before leaving the anchor zone. The second important performance metric is called availability. It is the fraction of nodes within an anchor zone possessing a piece of content. The success ratio depends on availability and increases in availability leads to increase in the success ratio.

In [4], the authors introduced a new zone called the Range of Interest (ROI). ROI is different from the anchor zone. Anchor zone acts as a replication zone for the content items; on the other hand, ROI is used to calculate different performance parameters like success ratio. The anchor zone can be much larger than ROI. The size of the anchor zone affects the success probability and availability. For example, the advertisement related to a special offer at a supermarket might be of interest to the people nearby so ROI can be set to let's say 300 meters. However, for achieving a high success probability, the anchor zone might be set much larger compared to ROI. Therefore, ROI can always be less than or equal to the size of the anchor zone.

The rest of the paper is organized as follows. Section II explains the latest related work regarding floating content. Section III presents some of the existing analytical models and simulation results that provide us with a deep insight about the performance evaluation of the floating content. In Section IV vital observations and discussions are presented. Section V concludes the paper.

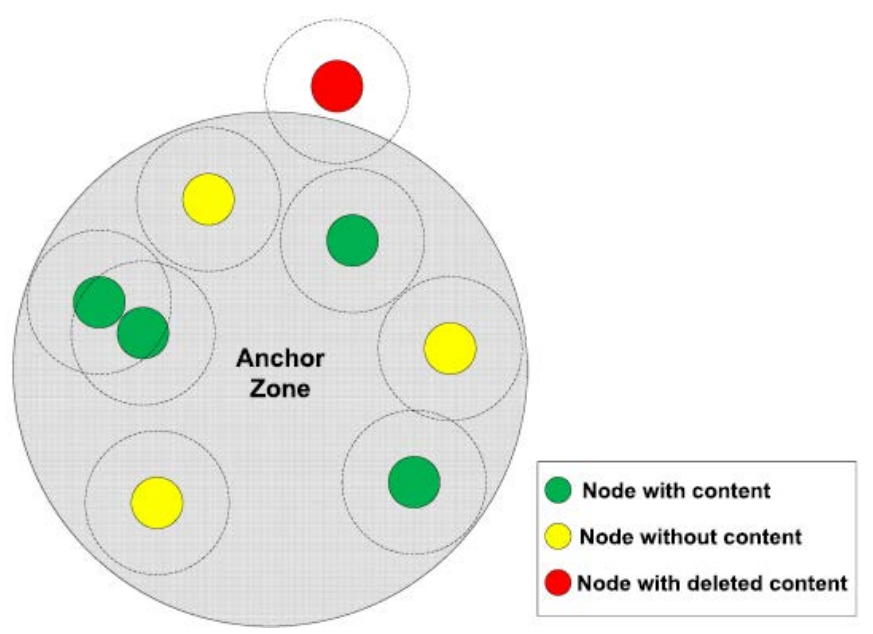

Fig. 1. Floating Content Replication Mechanism. 


\section{RELATED WORK}

After the first appearance of floating content in [3], various works have focused on different aspects of floating content. In [5], the authors confirmed that the analytical model presented in [4] can predict the success probability for Manhattan Grid Mobility model; however, for more complex models like Reference Point Group Mobility (RPGM) model and realistic vehicular traces, estimates were not quite accurate. As success probability is highly dependent upon the mobility characteristics of the users, therefore, analytical model capturing such characteristics was needed.

The first implementation of floating content as a communication service for a real-world application was done in [6]. An android based application called "Floaty" was developed and tested in an office environment. Bluetooth technology was used for communication among users. The experimental results confirmed the suitability of using floating content as a communication service in an office environment.

In [7], a modified version of the application used in [6] was used in an experiment in a university campus environment. It was shown that the mobility model in a university campus environment was quite different from the one that was observed in [6]. However, the results highlighted that a relatively low user density is enough to guarantee content persistence over time. This was contrarily to the predictions from the models presented in [3]. The experiment showed that even under a low user density, the content floats for a substantial time.

In [8], the authors proposed a new mobility model called Poisson Jumps mobility model. In this work the authors presented a new analytical model for the Poisson Jumps mobility model in a campus environment. The authors proved that Poisson Jumps mobility model can emulate the mobility patterns observed in a campus environment. The presented analytical model also captured the key performance metrics such as success probability and availability.

The performance of floating content is also investigated for VANETs. In [9], an analytical model was proposed for prediction of performance of the floating content in VANET environment. Instead of considering the road geometry, the analytical model was based on a modified version of the Random Waypoint mobility model. The proposed analytical model was evaluated under both synthetic and real-world vehicular traces. The model could predict performance accurately under both settings. The authors also conducted the performance analysis for floating content in a VANETs setting by proposing a new synthetic mobility model called District Mobility Model (DMM). An analytical model was developed based on DMM and performance of the floating content was evaluated under different mobility patterns and traffic conditions. The results proved the effectiveness of floating content under a wide range of traffic conditions.

In recent related work, it can be observed that the early works focused on the conditions under which a piece of content floats in an area. Later on, works focused on different analytical models and simulations for calculating different performance metrics. After confirming the persistence of the floating content from the analytical models, research focused on the real-world implementation of few applications using floating content as a communication service. Different analytical models were also developed for capturing the mobility characteristics in different environments like university campus and vehicular networks.

\section{ANALytical Models AND Simulations}

Various analytical models are presented in the literature regarding different performance parameters for floating content. These models vary based on the mobility of nodes ranging from simple mobility models [4] to relatively complex mobility models [7] [3] [8] incorporating synthetic traces and real-world mobility traces for the users. For this paper, a simple analytical model is considered, which is capable of predicting the performance of floating content for various parameters. Though this model was originally proposed for a simple mobility model called Random Direction mobility model, in [5] it was proven that despite its simplicity, it is capable of predicting performance for various relatively complex mobility models.

The analytical model presented in [4] provides an equation (equation 1) for calculating the success probability $P s$ under Random Direction Mobility model. Equation (1) has two parts. The first part represents the probability of meeting $k$ nodes along a trajectory within an anchor zone. This part is essentially dependent on the pure geometry and can be altered for different mobility models. The second part represents the probability that one of the $k$ nodes meets another node having the content and content replication take place.

$$
\begin{aligned}
& P s(\tau)= \\
& \int_{0}^{2 R} \frac{l^{2}}{\pi R^{2} \sqrt{4 R^{2}-l^{2}}} \cdot \sum_{k=1}^{\infty}[1- \\
& \left.\left(1-\frac{Q \bar{n}}{(\bar{m}+\bar{n})}\right)^{k}\right] \frac{\left(2 r \lambda\left(l^{\wedge} v \tau\right)\right)^{k} e^{-2 r \lambda\left(l^{\wedge} v \tau\right)}}{k !} d l
\end{aligned}
$$

The contribution of this analytical model is novel because it provides a simple (in that it uses few primitive system parameters) analytical model for computing success probability. This model was based on a simplistic mobility model called Random Direction mobility model. By extensive simulations, it was proven that the analytical model was capable of predicting the success probability for a wide range of system parameters.

The analytical model presented in [4] can be used to determine the size of the anchor zone required for getting 90 percent success probability for a given ROI. For instance, Fig. 2 presents the value of anchor zone required for a ROI equals to 300 meters for getting a success probability of 90 percent. It can be observed that a time comes when the anchor zone radius becomes equal to the radius of ROI for achieving a success probability of 90 percent. This is the condition under which the anchor zone radius and ROI are equal and anchor zone radius is sufficient for achieving 90 percent success probability. 


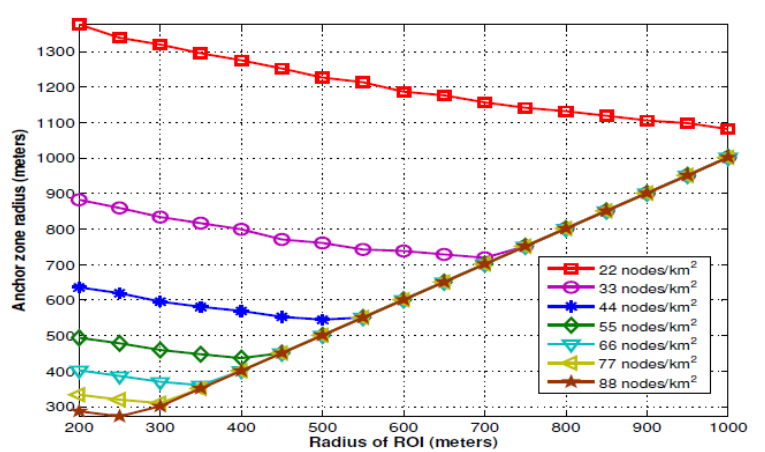

Fig. 2. Anchor Zone Radius for Achieving 90 Percent Success Probability.

Despite the simplicity of the analytical model, by doing extensive simulations in [5], it was observed that the approximates provided by the analytical model are close to a variety of mobility models including Manhattan Grid Mobility Model (MGMM), Reference Point Group Mobility (RPGM), and synthetic vehicular traces from Cologne city as shown in Fig. 3.

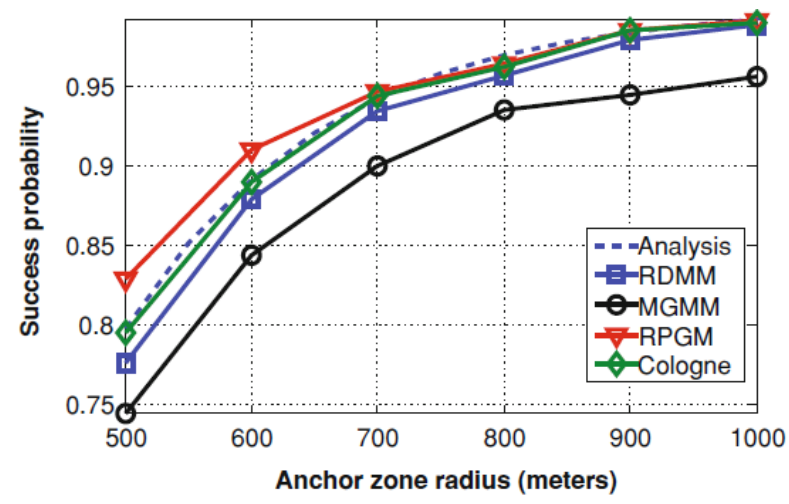

Fig. 3. Success Probability for different Mobility Models.

It is worth explaining that the communication model used by the model presented in [4], includes a parameter called ' $Q$ ' representing the probability of a successful transfer between two nodes when they communicate with each other.

The value of $Q$ equals to 1 means that there is a 100 percent success rate for an exchange of messages between two nodes. This parameter allows more complex communication models to be incorporated in the analytical model. This is one of the possible ways to extend the existing analytical model for different communication technologies such as Wifi-Direct and Bluetooth.

\section{OBSERVATIONS AND DISCUSSION}

This section presents some of the important observations that were observed during the course of this work.

\section{A. Impact of Communication Technologies on the Performance}

It is worth noting that for real-world implementation and performance evaluation of floating content, Bluetooth was used [6] [7]. One of the more prominent communication technologies based on pure ad hoc communication is WiFiDirect. It offers much longer communication range and much higher data transfer rates compared to Bluetooth [10].
Alternatively, for VANETs, a different standard known as 802.11p is available [9].

The bottom-line is that each communication technology has its own specific characteristics (like communication range, data transfer rate, etc.).

Therefore, first from the analytical model's perspective, it is very important to incorporate the specific characteristics of the communication technology into the model.

Second, from the practical (real-world implementation) perspective, it is important to utilize these different communication standards for developing the applications for doing the performance evaluation.

\section{B. Applications based on Floating Content}

Previously, the applications developed using floating content as the communication service [7] [6] [8] were experimental in nature and were restricted to different environments such as an office or a university campus.

The potential of floating content was realized recently in a few killer applications that surfaced particularly for postdisaster management and emergency situations [9].

In this paper, I would like to mention a recent application called "Tabaud" [11]. It is an application for both Android and Apple platforms and is initiated by the government of Saudi Arabia as a measure to contain the Corona virus (COVID-19).

This application utilizes Bluetooth for sending notifications to people if they are in the vicinity of an infected COVID-19 person. By using this application, a person can take essential precautions if he is in the vicinity of an infected person with COVID-19. This application has more than 2 million downloads on Android platform alone.

This application utilizes the communication service similar to floating content for exchanging messages among users who are within the vicinity of each other. The benefit of using Tabaud application is that even if a user is not connected to a fixed network like 4G or $\mathrm{Wi}-\mathrm{Fi}$, still he/she is able to receive alert notifications about a COVID19 infected person near him/her.

Similarly, applications based on the concept of Geo Fencing are also getting common. Geofencing is a concept in which a user in notified about an event/alert if he/she is in the vicinity of a particular location. Applications such as Checkmark 2, Geo Alert: location reminder, etc. are based on this concept.

There is a huge potential for using floating content as a communication service for communicating with users in the vicinity without relying on 3G/4G networks.

Similarly, there is a huge potential for using floating content as a communication service for the applications related to geographically restricted advertisements. For example, advertisements or special offers at a shopping mall might be of most interest to the people nearby. Floating content can prove to be very beneficial for such geographically restricted information dissemination. 


\section{Energy Consumption}

A crucial consideration while designing and implementing any application for smart devices is energy consumption due to the operation of that particular application.

As using floating content as a communication service requires periodically scanning for compatible devices within the vicinity, therefore it is important to investigate the energy consumption aspect as well.

An experiment was performed by running Floaty application developed in [8] and the results are shown in Fig. 4. In the experiment a total of 12 smartphones belonging to three different companies, i.e., HTC, Sony, and Samsung were present in the vicinity of each other.

All the smartphones were running the Floaty App, and Fig. 4 shows that on average each smartphone consumed between 3.5 and 4 percent battery per hour.

It is worth noting that optimizations for the application can be made by tuning in the different parameters like scan interval, message generation interval etc. However, for a normal user, this energy consumption seems high because after 12 hours of operation, the application would have consumed roughly 40 to 50 percent of battery.

Similarly, as Bluetooth was used by the Floaty app, therefore use of different communication technologies like Wifi-Direct is another open question that can be addressed in future work.

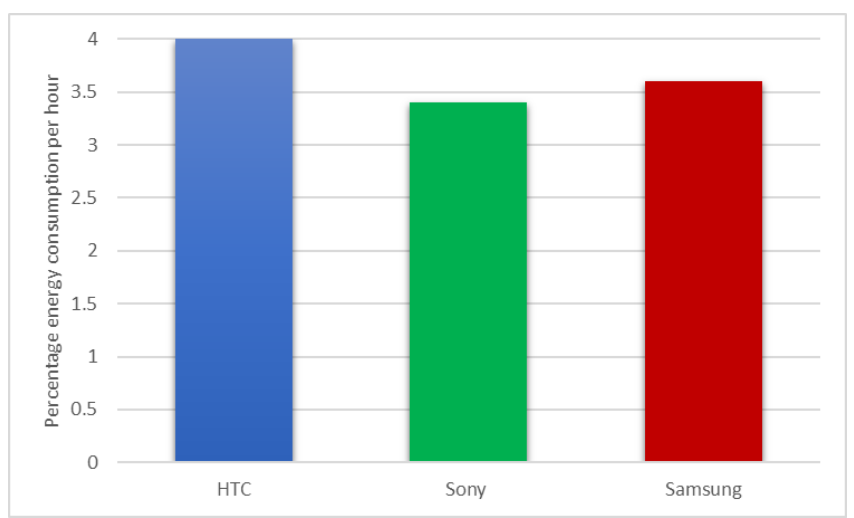

Fig. 4. Energy Consumption by an Application using Floating Content as Communication Service.

\section{CONCLUSION}

In this paper, the experiences learned from the recent research in a communication paradigm called floating content is presented. This work considered a simple analytical model and focused on the ways of extending this model for incorporating different communication technologies and mobility models. This paper also highlighted the impact of different communication technologies on the performance of floating content. Some of the existing applications using floating content were also illustrated and results related to the energy consumption of the smart devices running applications based on floating content were also discussed. Based on the discussions, new future directions were highlighted that can prove to be very beneficial for the researchers investigating in this area.

\section{REFERENCES}

[1] P. Rosenberger and D. Gerhard, "Context-awareness in industrial applications: definition, classification and use case," Procedia CIRP, vol. 72, pp. 1172-1177, 2018.

[2] A. A. V. Castro, G. D. M. Serugendo and D. Konstantas, "Hovering Information - Self-Organising Information that Finds Its Own Storage," in IEEE International Conference on Sensor Networks, Ubiquitous, and Trustworthy Computing, Taichung, 2008.

[3] E. Hyytiä, J. Virtamo, P. Lassila, J. Kangasharju and J. Ott, "When does content float? Characterizing availability of anchored information in opportunistic content sharing," in IEEE INFOCOM, Shanghai, 2011.

[4] S. Ali, G. Rizzo, B. Rengarajan and M. A. Marsan, "A simple approximate analysis of floating content for context-aware applications," in MobiHoc '13, Bangalore, 2013.

[5] S. Ali, G. Rizzo, V. Mancuso and M. A. Marsan, "Impact of Mobility on the Performance of Context-Aware Applications Using Floating Content," in International Conference on Context-Aware Systems and Applications, Viet Tri City, 2013.

[6] S. Ali, G. Rizzo, V. Mancuso, V. Cozzolino and M. A. Marsan, "Experimenting with floating content in an office setting," IEEE Communications Magazine, vol. 52, no. 6, pp. 49-54, 2014.

[7] S. Ali, G. Rizzo, V. Mancuso and M. . A. Marsan, "Persistence and availability of floating content in a campus environment," in IEEE Conference on Computer Communications (INFOCOM), Kowloon, 2015.

[8] G. A. Rizzo, V. Mancuso, S. Ali and M. A. Marsan, "Stop and forward: Opportunistic local information sharing under walking mobility," Ad Hoc Networks, vol. 78, pp. 54-72, 2018.

[9] G. Manzo, M. A. Marsan and G. Rizzo, "Performance Modeling of Vehicular Floating Content in Urban Settings," in International Teletraffic Congress (ITC 29), Genoa, 2017.

[10] S. Iskounen, T. Nguyen, S. Monnet and L. Hamidouche, "Device-toDevice communications using $\mathrm{Wi}-\mathrm{Fi}$ direct for dense wireless networks," in 7th International Conference on the Network of the Future, Buzios, 2016.

[11] Tabaud. [Online]. Available: https://play.google.com/store/apps/details? id=sa.gov.nic.tabaud. [Accessed 07 February 2021]. 